\title{
Hierarchic Fuzzy Approach Applied in the Development of an Autonomous Architecture for Mobile Agents
}

\author{
Márcio Mendonça ${ }^{1}$, Esdras Salgado da Silva ${ }^{1}$, Karina Assolari Takano ${ }^{1}$, \\ Mauricio Iwama Takano ${ }^{1}$, and Lúcia Valéria Ramos de Arruda ${ }^{2}$ \\ ${ }^{1}$ Postgraduate Mechanical Engineering Program (PPGEM) \\ Federal Technological University of Paraná (UTFPR) \\ Av. Alberto Carazzai, 1640, Centro, 86300-000, Cornélio Procópio, Paraná, Brazil \\ ${ }^{2}$ Postgraduate Eletrical Engineering And Industrial Computers Program (CPGEI) \\ Federal Technological University of Paraná (UTFPR) \\ Av. Sete de Setembro, 3165, Rebouças, 80230-901, Curitiba, Paraná, Brazil \\ \{mendonca, takano, 1vrarruda\} @utfpr.edu.br, \\ esdras.utfpr@bol.com.br, ka.assolari@gmail.com
}

\begin{abstract}
The development of a controller architecture based on hierarchic fuzzy logic to create the trajectory of a mobile agent in a virtual environment is initially used. The objective of the low cost controller is to regulate an opensource autonomous explorer robot, which moves between two pre-established points. The system establishes a viable route in a scenario with obstacles. Simulations in virtual environment are used to ensure the autonomy of the developed controller. Initial results demonstrate the success of this proposal.
\end{abstract}

Keywords: Autonomous agent, Fuzzy logic, Virtual kinematic model, Intelligent Systems, Hierarchic Fuzzy.

\section{Introduction}

Researches about mobile autonomous robots are becoming more important due to technological evolution and high processing computers. Some technological evolutions that can bear witness to are the development of intelligent computational techniques, such as fuzzy logic, artificial neural network, intelligent agents, and others.

A number of challenging problems must be solved for mobile autonomous navigation. The problems can depend on different domains like perception, localization, environment modeling, and decision making, control etc. [1].

Autonomous robots can be used in different fields for instance in high risk areas (to avoid risky situations for humans), in exploration of unattainable places of difficult access or hazardous environment, and in service robots which makes this an emergent field of research and application, as presented by [2, 3, and 4].

Explorer robots or agents can be virtually controlled by humans; this however demands communication systems, cameras and operators. A solution for this is the use of intelligent computational systems which can be applied in the development of robots with the ability of adaptation in different environments by reading signals from low level sensors such as ultrasound sensors [5]. This can be helpful in cases such as 
the exploration of natural environment (inhospitable) when autonomous decisions are required $[6,7,8]$.

The environment exploration has some difficulties to be solved for example uncertainties, inaccuracies and sequential errors. The complexity involved in creating an efficient path is known to be high due to sequential dynamic decisions that have to be made (a wrong decision can compromise the next steps). The navigation tasks are initiated by informing an objective. During the exploration the agent needs to have the ability to deal with unpredictable events like mobile and/or unexpected obstacles [9].

According to Khodayari [10] there are two main strategies to develop autonomous agents. The first one is related to heuristic knowledge and is represented in this paper by fuzzy logic as presented in $[11,12,13]$, and adapted to the model used. The second one is based in the control theory, subdivided in linear control theory and nonlinear control theory.

In past decades, researches have developed many obstacle avoidance navigation algorithms. These methods can be divided into the following categories: model-based method [14-19], fuzzy logic method [20-23] and reactive method based on neural network [24, 25]. Of all the methods, the obstacle avoidance method based on fuzzy logic reacts quicker and is less susceptible to sensor measuring errors for the robot's reaction has come from qualitative reasoning of different categories in the fuzzy logic method, so the algorithm proposed in this paper is based on fuzzy logic principles. The difference is the fact that the hierarchical fuzzy quantitatively simplify solving the problem by organizing rules with one fuzzy used for each objective.

The objective of this paper is to develop an autonomous explorer agent (robot) based in a low cost and open source platform with the ability to learn navigation strategies by environment interaction. The development proposal adopted is inspired by Braitenberg [26], who suggests the application of intelligent computational techniques starting up with a simple model with one or only a few functionalities, and gradually adding new objectives to improve the exploration capability of the agent, such as manage the battery charge and navigate back to the recharging spot. In this paper, the agent will have initially the ability to navigate through two pre-established points in a surface. The second objective is to add the ability to deviate from any obstacles that are found in the proposed initial path.

This paper is organized as follows: section 2 briefly describes the fuzzy logic concept; section 3 presents the development of this work (kinematic model for the robot position and controller); sections 4 and 5 show the results and conclusions.

\section{Fuzzy Logic Fundamentals}

Fuzzy systems according to Zadeh [27] is a set of methods based in the linguistic terms, multivalued logic, systems based in rules that aim to work with failure tolerance, inaccuracy and uncertainty in real systems. These methods and linguist terms proposes the concept of the fuzzy logic which consists of the combination of these theories, allowing the modeling of complex processes (based on inaccurate or approximate information) expressing control rules in linguistic terms. Different areas of knowledge use this technique as a solution for control applications, pattern recognition and function approximation, especially when there is little or no knowledge of the mathematical models. 
A fuzzy system consists of three main components: input and output, set of rules and inference mechanisms [28, 29]. A difficulty to implement simple Fuzzy logic is that as you increase the number of variables involved, the number of rules grow exponentially. One possible solution is the development of a fuzzy hierarchical structure in which different rule bases are used to address each goal [30].

\section{Development}

\subsection{Kinematic Model for the Robot Position}

There are two ways of representing the robots: kinematic or dynamic. The kinematic models are used to represent the robots according to the velocity and orientation of the wheels, and the dynamic models according to the generalized forces applied by the actuators. There are two kinematic models used to represent the agents: position model and configuration model. The first of them considers only the position and the orientation of the robot, while the second one also considers other internal variables (for example angular movement of the wheels). The position model fulfills the needs of the position control and spatial orientation of the robot. Most of the researches reported in the literature describe the robot according to Cartesian coordinates using just a position kinematic model [31, 32, and 33].

The robot used as a model in this paper consists of three wheels, one of which is mobile (in the front part of the robot) and the other two stationary on the sides (each of them connected to a motor); three ultrasonic sensors placed to collect signals that identify possible obstacles in the path. The physical model of this agent is shown in Fig. 1 (a), and the schematic model in Fig. 1 (b).

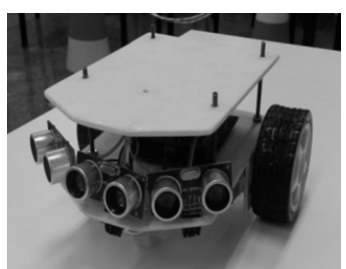

(a)

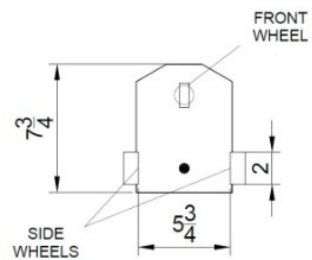

(b)

Fig. 1. Physical (a) and schematic (b) models of the robot

\subsection{Hierarchical Fuzzy Approach}

In some situations fuzzy logic using just a set of rules can control processes in different situations, during start up and course of operations with the proper activation of the rules. However when there are different objectives in the same controller, it is necessary to apply a set of rules for each objective.

Summarized when the fuzzy controller requires more complexity to achieve the desired goal, a common strategy is to decompose the controller problem hierarchically, using low-dimensional fuzzy systems. A priority is defined between the fuzzy systems adopted [34]. It is called Fuzzy hierarchical system using more than one fuzzy and establishes a hierarchy between them. 
A hierarchical fuzzy architecture based in cognitive architecture [34] especially the subsumption [35], was developed to work with hierarchical decision making and this is due to the need of two opposite main targets for example reach a target and deviate from obstacles in the trajectory.

The subsumption fuzzy architecture proposed has two sets of rules which work independently and with different and hierarchical competence levels. When certain competence level is enabled all other levels are disabled. Thereby the fuzzy controller coordinates the competence levels prioritizing the higher levels (deviate from obstacles is a higher level than reaching the target) [36]. In other words, when the autonomous agent is deviating from an obstacle it stops going after the target, this demands an implemented switching mechanism. In Fig. 2 it is shown the hierarchical fuzzy architecture developed.

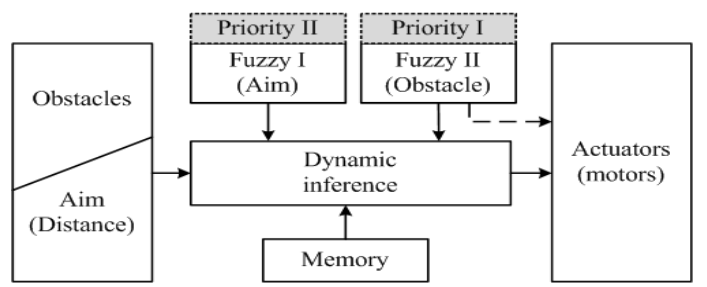

Fig. 2. Hierarchical model of the fuzzy system

Fuzzy I, the initial objective of this paper, uses a decision-making system based in heuristic methods. This step has the objective of determining the shortest route between the initial point and the target. This set of rules was implemented from observations of the dynamic behavior of the agent in virtual scenarios.

Table 1. Example of fuzzy rules I

1. If (LatDist is farLeft) and (FrontDist is farF) then (LeftPulse is me-
dium)(RightPulse is high)
2. If (LatDist is farLeft) and (FrontDist is mediumF) then (LeftPulse is
slow)( RightPulse is high)
3. If (LatDist is farLeft) and (FrontDist is closeF) then (LeftPulse is high)

This strategy consists in abstracting the input data (of mathematical simulations of the lateral and frontal distances) and converting them in linguistic variables (sets of rules) and then generating a response (pulses to the motors linked to the left and right wheels).

In Fig. 3 are presented the control surfaces of the fuzzy I corresponding to the left (Fig. 3a) and right (Fig. 3b) wheels. 


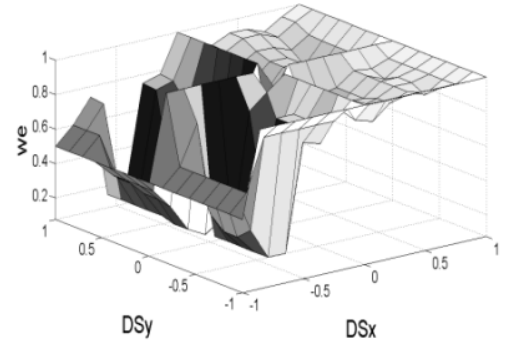

(a)

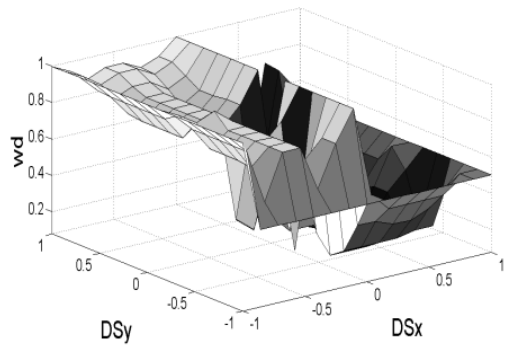

(b)

Fig. 3. Fuzzy I control surfaces for left (a) and right (b) wheels

The $\mathrm{x}$-axis represents the lateral distance between the robot and the target (DSx), the $y$-axis, the front distance (DSy) and the z-axis, the pulses wheels: right to left in Fig. 3a and Fig. 3b. Whenever the sensors detect an obstacle, the hierarchical fuzzy controller prioritizes the deviation of the obstacles using the fuzzy II set of rules. When no obstacle is detected, the agent will keep going after the target using the fuzzy I set of rules. Some of the rules used in fuzzy II are shown in table 2:

Table 2. Example of fuzzy rules II
1. If (sensor_L is far) and (sensor_F is far) then (LeftPulse is medium)
2. If (sensor_L is far) and (sensor_F is medium) then (LeftPulse is high)
3. If (sensor_L is far) and (sensor_F is close) then (LeftPulse is high)

The control surfaces related to the second fuzzy are presented in Fig. 4. Fig. 4a refers to the influence of both left and frontal sensors on the left wheel. Fig. $4 \mathrm{~b}$ refers to the influence of both right and frontal sensors on the left wheel. In Fig. 4b, "we" represents the pulses on the left wheel with range [0.14 0.19] because when we have obstacles on the right hand front of the robot, it has to turn to left. Therefore there is a low pulse in left wheel. The rules are symmetric, which means the results are the same for the right wheel.

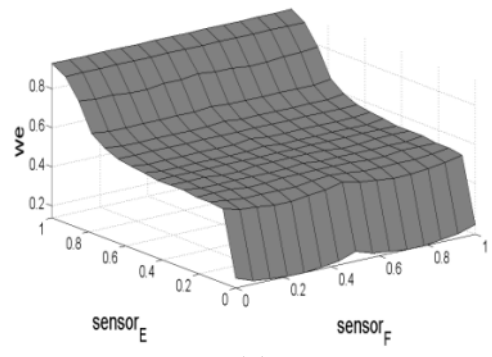

(a)

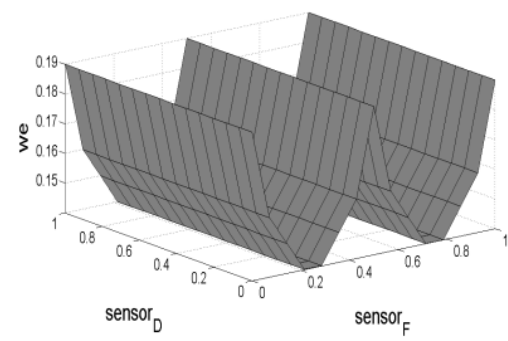

(b)

Fig. 4. Fuzzy II control surfaces for left wheel 
A two dimensional simulator was implemented in Matlab to study the dynamic behavior of the mobile agent. The scale used for both the prototype and the scenario is $1: 100$.

Russel and Norvig [06] suggest that in order to consider an autonomous agent, it is necessary to succeed in at least three different simulations. Therefore the arrangement of the obstacles, the initial position of the mobile agent and the target are adjustable in the Matlab program, allowing the construction of different scenarios.

In the simulator developed, the sensors were calibrated to send the signals at a maximum distance of $15 \mathrm{~cm}$.

\section{$4 \quad$ Results}

For each scenario simulated, three situations where considered (except 4): scenario with obstacles; rearrangement of these obstacles; and scenario with noise of five percent on the sensors signals. The noise added in the sensors signals represents the uncertainty of the data acquisition, characteristic of real scenarios due to different behavior of the sound wave reflection in different materials.

In the following subsections are presented the simulated scenarios. In the following images, items (a) and (b) indicate tests in obstacles arranged in two different ways, and item (c) indicates the response to noises.

\subsection{Scenario 01}

In this scenario (Fig. 5) the initial position for the prototype is $(0,0)$, at an angle of zero degrees with the $\mathrm{X}$-axis, and the target is located in position $(50,70)$.

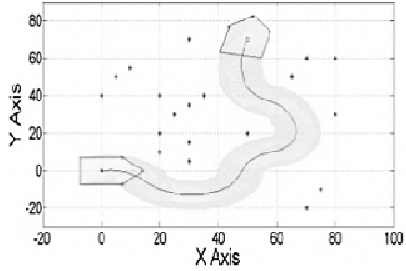

(a)

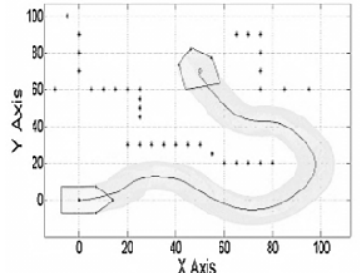

(b)

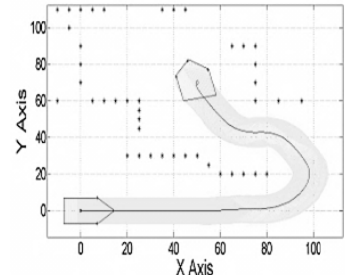

(c)

Fig. 5. Scenario 01

\subsection{Scenario 02}

In this scenario (Fig. 6) the initial position for the prototype is $(30,25)$, at an angle of 45 degrees with the $\mathrm{X}$-axis, and the target is located in position $(-50,80)$. 


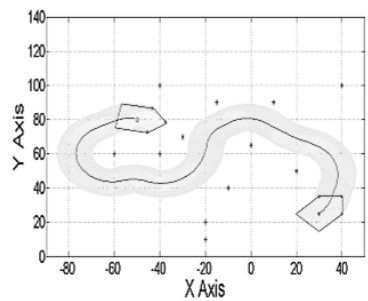

(a)

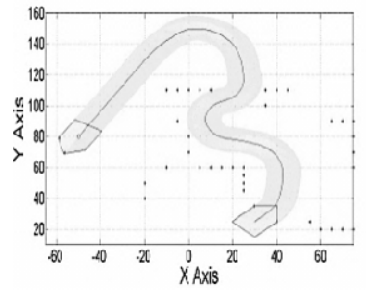

(b)

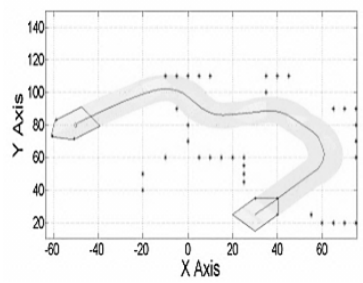

(c)

Fig. 6. Scenario 02

\subsection{Scenario 03}

In this scenario (Fig. 7) the initial position for the prototype is $(-80,75)$, at an angle of zero degrees with the $\mathrm{X}$-axis, and the target is located in position $(35,75)$.

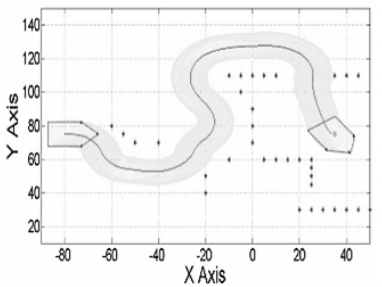

(a)

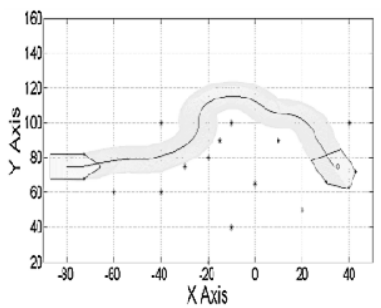

(b)

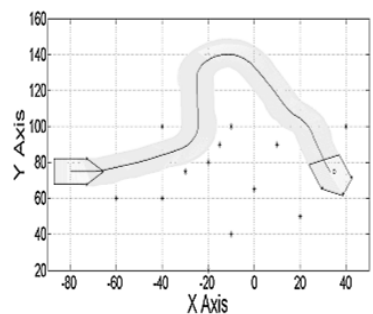

(c)

Fig. 7. Scenario 03

\subsection{Scenario 04}

In this scenario (Fig. 8) the initial position for the prototype is $(-50,-90)$, at an angle of zero degrees with the $X$ axis, and the target is located in position $(0,0)$. In this simulation was considered the complexity of a spiral scenario. In this scenario situation (b) was not considered.

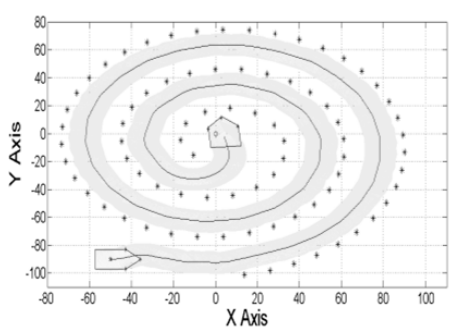

(a)

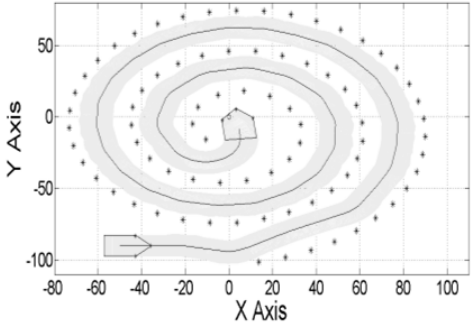

(c)

Fig. 8. Scenario 04 


\section{Conclusion}

The initial results obtained from the simulations were convincing, because the mobile agent accomplished the goal of reaching the target with a maximum error of one centimeter deviating from obstacles.

It is possible to reduce the error. However, it would make the robot moves more than necessary if an obstacle is located at about ten centimeters of the target. This happens because of the sensors that identify the obstacle and do not allow the agent to reach the target, as the hierarchy prioritizes the deviation of the obstacles.

Seven different scenarios were successfully simulated. This implies the autonomy of the proposed fuzzy control. The simulations with uncertainties also imply a robust system, because they approach simulations to real environment where data input from the sensors may have noise and errors. Future works are presented:

- Improve the minimum error by changing the hierarchical process;

- Compare the proposed controller with other intelligent techniques like Artificial Neural Network and Fuzzy Cognitive Maps;

- Genetic algorithm can also be used to tune the fuzzy logic controller;

- Improve the complexity of the scenarios using for example, walls;

- Add new functions in the controller: battery management (locate charging points) and reverse gear for extreme situations;

- Apply the system in a real robot using an open source development platform, such as a low cost microcontroller (example, Arduino).

Acknowledgment. The authors would like to thank CNPq and Araucaria Foundation of the State of Paraná, Brazil, for financial support.

\section{References}

1. Shaikh, M.H., Kosuri, K., Ansari, N.A., Khan, M.J.: The state-of-the-art intelligent navigational system for monitoring in mobile autonomous robot. In: 2013 International Conference on Information and Communication Technology (ICoICT), pp. 405-409, 20-22 (2013)

2. Asami, S.: Robots in Japan: Present and Future. IEEE Robotics and Automation Magazine 1(2), 22-26 (1994)

3. Schraff, R.: Mechatronics and Robotics for Service Apliccations. IEEE Robotics and Automation Magazine 1(4), 31-35 (1994)

4. Fracasso, P.T., Costa, A.H.R.: Navigation From Reactive Autonomous Mobile Robots Using Fuzzy Logic Rules With Weighted. SBAI (2005)

5. Mendonça, M., Arruda, L.V.R., Neves Jr., F.: Autonomous navigation system using Event Driven-Fuzzy Cognitive Maps. Applied Intelligence 37(2), 175-188 (2011)

6. Russell, S.J., Norvig, P.: Artificial Intelligence: A Modern Approach. Prentice Hall, Englewood Cliffs (1995)

7. Calvo, R., Romero, R.A.F.: A Hierarchical self-organizing controller for navigation of mobile robots. In: Proceedings of International Joint Conference on Neural Netwoks. IEEE World Congress Computational Intelligence, Vancouver (2006) 
8. Broggi, A., Zelinsky, A., Parent, M.E., Thorpe, C.E.: Intelligent vehicles. Springer Handbook of Robotics, pp. 1175-1198 (2008)

9. Bakambu, J.N.: Integrated autonomous system for exploration and navigation in underground mines. In: Proc. 2006 IEEE/RSJ Int. Conf. Intelligent Robots and Systems, pp. 2308-2313 (2006)

10. Khodayari, A.G.: A historical review on lateral and longitudinal control of autonomous vehicle motions. In: 2nd International Conference on Mechanical and Electrical Technology (ICMET), Singapore (2010)

11. Chiang, H.-H., Ma, L.-S., Perng, J.-W., Wu, B.-F.: Longitudinal and lateral fuzzy control systems design for intelligent vehicles. In: Proceedings of the 2006 IEEE International Conference on Networking, Sensing and Control (ICNSC), pp. 544-549 (2006)

12. Yang, J.: e Zheng, N.: An expert fuzzy controller for vehicle lateral control. In: 33rd Annual Conference of the IEEE on Industrial Electronics Society (IECON), pp. 880-885 (2007)

13. Cai, L., Rad, A.E., Chan, W.-L.: An intelligent longitudinal controller for application semiautonomous vehicles. IEEE Transactions on Industrial Electronics 57(4), 1487-1497 (2010)

14. Lim, J.H., Cho, D.W.: Sonar based systematic exploration method for an autonomous mobile robot operating in an unknown environment. Robotic (16), 659-667 (1998)

15. Krishna, K.M., Kalra, P.K.: Perception and remembrance of the environment during realtime navigation of a mobile robot. Robotics and Autonomous Systems (37), 25-51 (2001)

16. Maaref, H., Barret, C.: Sensor-based navigation of a mobile robot in an indoor environment. Robotics and Autonomous Systems (38), 1-18 (2002)

17. Chatterjee, R., Matsuno, F.: Use of single side reex for autonomous navigation of mobile robots in unknown environments. Robotics and Autonomous Systems 35, 77-96 (2001)

18. Ge, S.S., Xuecheng, L., Al Mamun, A.: Boundary following and globally convergent path planning using instant goals. IEEE Trans. on Systems, Man, and Cybernetics-Part B: Cybernetics 35(2), 240-254 (2005)

19. Toibero, J.M., Carelli, R., Kuchen, B.: Switching Control of Mobile Robots for Autonomous Navigation in Unknown Environments. In: IEEE International Conference on Robotics and Automation, Roma, Italy, pp. 1974-1979 (2007)

20. Xu, W.L., Tso, S.K.: Sensor-based fuzzy reactive navigation of a mobile robot through local target switching. IEEE Trans. Syst., Man Cybern., C 29(3), 451-459 (1999)

21. John, H.L.: Evolution of a Negative-Rule Fuzzy Obstacle Avoidance Controller for an Autonomous Vehicle. IEEE Trans. on Fuzzy Systems 15(4), 718-727 (2007)

22. Chao, C.-H., Hsueh, B.-Y., Hsiao, M.-Y., et al.: Fuzzy Target Tracking and Obstacle Avoidance of Mobile Robots with a Stereo Vision System. International Journal of Fuzzy Systems 11(3), 183-191 (2009)

23. Abiyev, R., Ibrahim, D., Erin, B.: Navigation of mobile robots in the presence of obstacles. Advances in Engineering Softwares 41, 1179-1186 (2010)

24. Jackson Phinni, M., Sudheer, A.P., RamaKrishna, M., et al.: Obstacle Avoidance of a wheeled mobile robot: A Genetic-neuro-fuzzy approach. In: IISc Centenary - International Conference on Advances in Mechanical Engineering, Bangalore, India, pp. 1-3 (2008)

25. Zhu, A., Simon, X.Y.: Neurofuzzy-Based Approach to Mobile Robot Navigation in Unknown Environments. IEEE Trans. on Systems, Man, and Cybernetics-Part C: Cybernetics 37(4), 610-621 (2007)

26. Braitenberg, V.: Vehicles: Experiments in Synthetic Psychology. The MIT Press (1986)

27. Zadeh, L.A.: Fuzzy algorithms. Info. \& Ctl. 12, 94-102 (1968)

28. Passino, M.K., Yourkovich, S.: Fuzzy control. Addison-Wesley, Menlo Park (1997) 
29. Kasabov, N.K.: Foundations of Neural Networks, Fuzzy Systems, and Knowledge Engineering. MIT Press (1998)

30. Wang, L.: Analysis and design of hierarchical fuzzy systems. IEEE Trans. Fuzzy Syst. 7, 617-624 (1999)

31. Bloch, A.M., Reyhanoglu, M., McClamroch, N.H.: Control and stabilization of nonholonomic dynamic sytems. IEEE Transactions on Automatic Control 37(11), 1746-1756 (1992)

32. Kolmanovsky, I., McClamroch, N.H.: Developments in nonholonomic control problems. IEEE Control Systems Magazine 15(6), 20-36 (1995)

33. Murata, S., Hirose, T.: On board locating system using real-time image processing for a self-navigating vehicle. IEEE Transactions on Industrial Electronics 40(1), 145-153 (1993)

34. Brooks, R.: A robust layered control system for a mobile robot. IEEE Journal of Robotics and Automation 2(1), 14-23 (1986)

35. Coppin, B.: Artificial Intelligence Illuminated. Jones And Bartlett Publishers, Burlington (2004)

36. Goerick, C.: Towards an understanding of hierarchical architectures. IEEE Trans. Auton. Ment. Dev. 3, 54-63 (2011) 